\title{
Treinta años de Alma-Ata: Interpretaciones y consecuencias para el bienestar psicosocial y la salud comunitaria
}

\author{
Isabel Aguilar Palacio \\ Instituto Aragonés de Ciencias de la Salud, Zaragoza (España) \\ Recibido: 7 de abril del 2009 / Aprobado: 24 de julio del 2009
}

En este artículo se discute la experiencia de treinta años, desde que se presentó, en 1978, en la Conferencia Internacional de Alma-Ata, la nueva estrategia de la OMS de Atención Primaria de Salud. En esta, la OMS apostaba por un sistema integral basado en la equidad y en la participación comunitaria. Esta estrategia fue tachada de utópica, desarrollándose distintas interpretaciones de un mismo concepto, entre ellas, la Atención Primaria de Salud (APS) y la Atención Primaria Selectiva de Salud (APSS). Ambos enfoques de la Atención Primaria han funcionado paralelamente pero de forma irreconciliable. Sin embargo, se sostiene que la efectividad del modelo APS ha quedado demostrada en la experiencia de su aplicación en varios países, donde se ha reducido la mortandad infantil y promovido la prevención.

atención primaria de salud / atención primaria selectiva de salud / salud comunitaria

Thirty years of Alma-Ata: interpretations and consequences for psychosocial well-being and communitarian health

In this article the experience of over 30 years since the presentation of a new strategy for Primary Health Care by the WHO in the International Conferencie meeting of Alma-Ata was discussed. This strategy was a comprehensive system based on equity and community participation. This initiative was accused of utopian, and several interpretations of the same concept arose, such as Primary Health Care (PHC) and Selective Primary Health Care (SPHC). Both interpretations have worked in a parallel but irreconcilable ways. However, it is argued for the efectiveness of the PHC demostrated in the application experience with the reduction of infant mortality and the promotion of prevention policies.

primary health care / selective primary health care / community health

Correo electrónico: Isabel.aguilarp@gmail.com 
Many social evolutions and revolutions have taken place because the social structures were crumbling. There are signs that the scientific and technical structures of public health are also crumbling.

MAHLER, 1975.

\section{INTRODUCCIÓN}

El concepto de salud, pese a la conocida definición de la OMS como estado de completo bienestar físico, psicológico y social, ha sido relegado a un plano puramente asistencial, con la progresiva medicalización del concepto de salud.

Esta medicalización que, a su vez, lo es social, enfatiza la responsabilidad del individuo en su propio nivel de salud (Barriga, 1993) mientras que, paradójicamente, las decisiones sanitarias son asumidas por organismos muchas veces ajenos a los deseos del ciudadano, en los que la comunidad no tiene capacidad de decisión.

Pese a la existencia de este modelo, han pasado ya más de treinta años desde que la OMS plantease su modelo de atención primaria de salud (APS) con el lema "salud para todos en el año 2000". Este modelo sanitario tenía como principal objetivo responder a las necesidades del individuo, así como a las demandas de la comunidad, siendo la respuesta de la OMS a una serie de necesidades en salud que se venían percibiendo y que generaron un cambio en el planteamiento de la salud: un auténtico abordaje biopsicosocial.

\section{LA OMS Y LA ATENCIÓN PRIMARIA DE SALUD}

En los años sesenta, tras el fracaso del programa de erradicación de la malaria, la OMS decide hacer una revisión de los servicios básicos de salud. Mientras que en la década de los cincuenta se pensó que el fin de la enfermedad era posible si el conocimiento y la tecnología sanitaria eran accesibles para todos (Van Balen, 2004), ahora la continuidad por parte de los sistemas de salud de los países receptores se considera una pieza clave en el éxito de dichos programas (Newell, 1988).

Influenciada por la Christian Medical Commission ${ }^{1}$ (CMC) con la que cooperarán en 1973 (Litsios, 2004) los "médicos descalzos" de la China comunista $^{2}$ y los movimientos antiimperialistas y de izquierda, la OMS desarrolló una estrategia de mejora integral de los sistemas sanitarios: al informe de los años sesenta sobre los sistemas básicos de salud le seguirá el de 1972 acerca del fortalecimiento de dichos servicios, el informe de 1975 sobre alternativas para necesidades básicas en países en desarrollo y otros escritos como "Health by

1 Cuerpo encargado por el Concilio Mundial de Iglesias de la evaluación de sus programas sanitarios en países en desarrollo. En 1970, en el periódico Contact, agrupan sus conclusiones en una serie de medidas a las que denominan "Comprehensive Health Care".

2 Sistema de trabajadores sociales en las zonas rurales, con acción preventiva y curativa. 
the people" de Newell. ${ }^{3}$ En estos informes se analizan los resultados favorables de políticas sanitarias de países como Bangladesh, China, Cuba, India o Níger (Cueto, 2004).

Finalmente, en 1975, la OMS agrupa sus conclusiones en una estrategia a la que da el nombre de "Primary Health Care"4 (Atención Primaria de Salud, APS) caracterizada por los siguientes principios:

- La APS debe ser una parte integral de los sistemas de salud, con servicios eficaces, aceptables culturalmente y asequibles dependiendo de los recursos de cada lugar.

- Debe proporcionar cobertura universal, con la posibilidad de llegar a poblaciones periféricas por medio de los health workers (personal preparado para realizar sencillas labo-

3 En este artículo, editado en 1975, Newell da ejemplos de países con sistemas basados en la comunidad: China, con los "médicos descalzos"; Venezuela, con su "simplified medicine", donde auxiliares de enfermería con formación preventiva y curativa se ocupaban de zonas rurales que carecían de médicos; y el caso de Tanzania, que movilizaba a su población rural en "Ujamaa villages" (estructuras socialistas diseñadas para incentivar la participación popular en desarrollo).

4 Como explica Tejada de Rivero (2003), el término se deformará. Para empezar, el original "Care" debería ser interpretado como "cuidado", ya que denota una acción de carácter horizontal, mientras que la "atención" es algo vertical, asimétrico y nunca participativo. En cuanto a "primario", en vez de considerarse como algo principal, se percibió como primitivo o poco civilizado. res preventivas y curativas ante la falta de personal médico).

- Las actividades desarrolladas por la APS son las de prevención, promoción, curación y rehabilitación.

- Las políticas desarrolladas en el ámbito sanitario deberán ir íntimamente ligadas a las de otros sectores del desarrollo.

- La participación comunitaria será un componente esencial, tanto para la formulación de necesidades como para el desarrollo de las actividades en salud, fomentando el diálogo entre la comunidad y los servicios (OMS, 1991).

Pese a que no se trataba de una idea nueva (Mahler, 1978) la APS adquirió relevancia por la celebración de la Conferencia Internacional de 1978 en Alma-Ata (actual Almaty, en Kazajstán) organizada conjuntamente por la OMS y la Unicef y a la cual asistieron 134 países, 67 organismos internacionales y organizaciones no gubernamentales, con China como la gran ausente.

\section{DE UNA IDEA A UNA CONFERENCIA:}

\section{EL SIGNIFICADO DE ALMA-ATA}

La celebración de la Conferencia Internacional sobre APS fue la consecuencia de una serie de intereses políticos, en el contexto de una situación internacional turbulenta, en plena Guerra Fría y con un bloque comunista dividido entre sus dos grandes potencias: China y 
la Unión de Repúblicas Socialistas Soviéticas (URSS).

Desde un principio, los soviéticos deseaban organizar una conferencia internacional sobre APS. Venediktov, el delegado de la URSS en la OMS, plantea esta cuestión en $1972,{ }^{5}$ encontrándose con la oposición del entonces presidente de la OMS, H. Mahler, quien creía que no era el momento oportuno, ya que confiaba en una acción entre oficinas regionales y subregionales como el método más fiable para presentar alternativas locales. ${ }^{6}$

Pese a las reticencias de Mahler, en marzo de 1976 se acepta realizar dicha conferencia (Litsios, 2002), que se 1levará a cabo dos años después en Kazajstán. ${ }^{7}$ Sin embargo, Mahler consiguió que lo presentado en Alma-Ata repre-

5 Había sido la URSS la que, en 1970, había presentado la resolución sobre desarrollo de los sistemas de salud.

6 Uno de los principios de la APS es que no existe un "sistema estándar", sino que los servicios de salud deben adaptarse a cada lugar. Además, Mahler no compartía el modelo soviético de APS, al considerarlo demasiado medicalizado y centralizado, lo cual permitía pocos cambios desde la comunidad. Por último, Mahler era consciente de que no era una conferencia internacional lo que proporcionaría un intercambio en aras de la cooperación.

7 El proceso de elección de sede tampoco fue sencillo. La OMS buscaba un país en desarrollo, ofreciéndose Egipto, pero renunciando posteriormente por la presión soviética. También lo hizo Costa Rica, con el apoyo de Estados Unidos, pero en este caso fue rechazado por la falta de infraestructuras. Finalmente, la URSS logró lo que se pro- sentase más su punto de vista que el soviético (Brown, Cueto \& Fee, 2006).

La relevancia de Alma-Ata no se debe tan solo a su amplia participación sino, sobre todo, porque abordó una cuestión que todavía no había sido tratada en profundidad: la inequidad en salud y en servicios sanitarios (Hall \& Taylor, 2003).

Esta estrategia integral fue presentada bajo el eslogan de "Salud para todos en el año 2000", en el que jugó un papel importante el nuevo concepto de salud $^{8}$ y donde se intentaba promover un compromiso político, pero también un cambio en las mentalidades personales. En palabras de Mahler:

This challenge is aimed focusing world attention on the grave inequities that exist and on the possibility of attaining an aceptable level of health, equitably distributed through the world in one generation's time [...] a realistic goal only if urgent action is initiated now (Mahler, 1978, p. 113).

Para el año 2000, los países deberían haber diseñado sus estrategias políticas con este fin y estar en un claro

ponía, favorecido por su aporte económico a la conferencia, pero con la negativa de la OMS a que se realizase en Moscú, de ahí la elección de Kazajstán.

8 Salud entendida como el estado de completo bienestar físico, psíquico y social, y no solo la ausencia de enfermedad, lo que marca los límites de la APS mucho más allá de la simple acción curativa. 
Tabla 1

Declaración de Alma-Ata (1978)

1. La salud es un derecho universal cuya consecución requiere de la interacción con otros sectores del desarrollo.

2. La inequidad en salud es inaceptable.

3. Salud y desarrollo van unidos en un binomio indisoluble.

4. La comunidad tiene el derecho y el deber de participar tanto en la creación de los programas de salud como en su realización.

5. El objetivo de los países debe de ser que para el año 2000 exista un nivel de salud que permita llevar una vida productiva en lo social y económico, lo cual será posible con un mejor aprovechamiento de los recursos.

6. Para conseguir dichos objetivos, la estrategia a seguir es la APS, la cual precisa de la cooperación entre países.

proceso de ejecución de medidas concretas.

No obstante, y pese a ser aprobada por unanimidad, ${ }^{9}$ la conferencia de Alma-Ata no tendría los resultados esperados.

\section{CONSECUENCIAS E INTERPRETACIONES: LA ATENCIÓN PRIMARIA SELECTIVA DE SALUD}

Tras la conferencia de Alma-Ata, los países firmantes adoptaron distintas estrategias:

- Legislación: La declaración se incorporó en la legislación de distintos países, siguiendo cuatro líneas:

9 De hecho la idea ya había sido "vendida" con anterioridad en una serie de campañas contrarreloj en las oficinas regionales de la OMS.
- Desarrollo de la APS en continuidad con los programas ya existentes, por ejemplo en Cuba y en zonas rurales de China.

- Adaptación a partir de los sistemas existentes, desarrollado en los Países Bajos.

- Innovación: países que optaron por crear una nueva estructura, como es el caso de España, ${ }^{10}$ que desarrolló su actual sistema de APS (Gervás, Pérez-Fernández, Palomo-Cobos \& PastorSánchez, 2005).

10 Se creó la especialidad de Medicina de Familia dentro del sistema MIR y las Unidades Docentes, y se establecieron las Zonas de Salud, los Centros de Salud y los Equipos de Atención Primaria en el marco del Real Decreto de 1984 sobre Estructuras Básicas de Salud. 
- Importación: al mundo de la cooperación, por medio de ONG $\mathrm{y}$ agencias de desarrollo.

- Negación: No se llevaron a cabo medidas concretas para llevar la declaración a la práctica (De Maeseneer, Williams, De Sutter, Van de Geuchte \& Billings, 2007).

Sin embargo, y por norma general, la estrategia presentada en Alma-Ata fue muy pronto criticada. Se habló de ella como una revolución socioeconómica que convertía a la OMS en una organización política. La APS fue considerada una atención de segunda clase para los pobres. Por parte de los profesionales se la tachó de simplista, antiintelectual y poco basada en la evidencia científica, que requería demasiados sacrificios personales, entre ellos la pérdida de privilegios.

La principal alternativa a Alma-Ata se desarrolló tan solo un año después, en 1979, en la conferencia de "Health and Population in Development" en Bellagio, organizada por la Fundación Rockefeller y con la participación de la Fundación Ford, el Consejo de la Población y el Banco Mundial (Fort, Mercer \& Gish, 2006).

Esta conferencia se basaba en un artículo publicado por Walsh y Warren en el que se acuñaba el término de Atención Primaria Selectiva de Salud (APSS). Estos autores defendían que los problemas no pueden ser afrontados de manera simultánea, por lo que es necesario establecer prioridades. ${ }^{11}$ Una vez establecidas, se elegirían las intervenciones más costo-efectivas (Walsh \& Warren, 1979).

Lo que realmente hizo que la APSS adquiriese relevancia fue la adopción por parte de la Unicef, en 1982, de la "Children's Revolution". En ella, y siguiendo el modelo de la APSS, adopta como estrategia para aumentar la supervivencia infantil una serie de medidas costo-efectivas, a las que se les dio el acrónimo de "GOBI", ${ }^{12}$ y que posteriormente se añadiría "FFF". ${ }^{13}$ Esto se tradujo en una serie de programas verticales a los cuales se adhirieron muchas organizaciones.

Así, unos pocos años después de la presentación de la APS nos encontramos con que la mayoría de las agencias internacionales apoyaban los programas selectivos. Esto provocaría un cisma entre dos formas de entender las políticas sanitarias.

11 Para establecer estas prioridades, los autores clasificaban las principales enfermedades de los países en desarrollo conforme a cuatro criterios: prevalencia, mortalidad, morbilidad y posibilidades de control, asignándoles una puntuación. Con ello, formaban tres categorías: enfermedades con alta, media y baja prioridad. Entre las de alta prioridad se seleccionaban posteriormente las que tenían un coste razonable y fuesen factibles.

12 Lo cual no deja de resultar paradójico, ya que tan solo cuatro años antes la Unicef había organizado la Conferencia de Alma-Ata junto con la OMS.

13 Growth monitoring, oral rehydratation, breast feeding and immunization. 


\section{APS Y APSS: ¿̇DOS MODELOS IRRECONCILIABLES O COMPLEMENTARIOS?}

Pese a que desde el principio los defensores de la APSS argumentaron que esta se trataba de una "medida interina" y que en ningún modo excluía a otras políticas sanitarias (Warren, 1988), muchos recursos se desviaron desde los programas de APS hacia los de la APSS. Eso, unido a una aproximación radicalmente distinta a los problemas de salud de las poblaciones, hizo que, aunque el fin fuese el mismo, el conflicto resultase inevitable.

Aunque por parte de los defensores de la APSS se afirmaba que la APS era la mejor estrategia para adoptar, se consideraba que no resultaba factible, debido a su alto coste y a la cantidad de personal que requería. De la misma opinión es el Banco Mundial, el cual, en su informe de 1993 (World Bank, 1993) reconoce el binomio salud y desarrollo, y apoya las medidas costoefectivas gestionadas por agencias donantes para reconducir los sistemas sanitarios de los países en desarrollo.

Muchas instituciones convinieron en la necesidad de concentrar los recursos en un número mínimo de intervenciones de probada eficacia, en contraposición a la propuesta de la APS, tachada en numerosas ocasiones de tener unos sistemas de evaluación y análisis débiles (Hall \& Taylor, 2003) y de no responder a tres cuestiones básicas: qué había que hacer, cómo debía reali-

Tabla 2

Diferencias entre la Atención Primaria de Salud (APS) y la Atención Primaria Selectiva de Salud (APSS)

\begin{tabular}{ll}
\hline \multicolumn{1}{c}{ APS } & \multicolumn{1}{c}{ APSS } \\
\hline $\begin{array}{l}\text { Estrategia integral, con programas } \\
\text { down-top que nacen de la comunidad. }\end{array}$ & $\begin{array}{l}\text { Programas verticales top-down basa- } \\
\text { dos en enfermedades concretas. }\end{array}$ \\
\hline $\begin{array}{l}\text { Participación de la comunidad, que } \\
\text { define sus propias necesidades en } \\
\text { salud. }\end{array}$ & $\begin{array}{l}\text { No participación comunitaria: medidas } \\
\text { planificadas por expertos externos. }\end{array}$ \\
\hline $\begin{array}{l}\text { Método de financiación no claro. } \\
\text { Metodológicamente débil. }\end{array}$ & $\begin{array}{l}\text { Financiada por agencias donantes. } \\
\text { dencia científica. }\end{array}$ \\
\hline $\begin{array}{l}\text { Intervención basada en el contexto } \\
\text { social. }\end{array}$ & Ignora el contexto social. \\
\hline
\end{tabular}


zarse y cuánto iba a costar (Cueto, 2004).

Estas carencias metodológicas, unidas a la consideración utópica del lema "Salud para todos en el año 2000", hicieron que las principales agencias donantes prefiriesen invertir en la APSS y en sus programas, los cuales proporcionaban garantías a corto plazo.

Será esta búsqueda del "éxito inmediato" uno de los aspectos más criticados por los defensores de la APS, tanto por el desvío de fondos que eso suponía como por sus efectos dañinos para la evolución hacia un sistema integrado: iniciativas locales satisfactorias se implantaban pronto en el ámbito nacional y viceversa, y no se realizaba la adaptación de los servicios de salud a cada lugar (Van Balen, 2004).

Otros muchos aspectos serán considerados como inaceptables por parte de los defensores de la APS:

- Reducción de los principios de Alma-Ata a una serie de intervenciones tecnológicas de alta prioridad, lo que supone la vuelta a los programas verticales.

- Falta de interés por el desarrollo de las comunidades, ${ }^{14}$ olvidando la base social de la enfermedad.

- No son las comunidades las que fijan sus prioridades en salud, sino

14 Family planning, female literacy and food supplementation. expertos dependientes de las agencias donantes, existiendo una imposición externa ${ }^{15} \mathrm{y}$, en muchas ocasiones, un conflicto de intereses, ${ }^{16}$ como en el caso de los programas de vacunación (Fleck, 2002).

- La APSS crea programas sanitarios de segunda clase, destinados a los estratos periféricos más pobres, y en muchos casos limitados a hospitales urbanos (Magnussen, Ehiri \& Jolly, 2004).

Pese a todo, los defensores de la APSS tomaron una actitud conciliadora y presentaron ambos modelos como complementarios. Así, argumentan que lejos de competir, los programas específicos son un medio de promover la participación de la comunidad y fortalecer los sistemas de salud. Un ejemplo de ello sería el Integrated Management of Childhood Illness Initiative, donde programas específicos incorporaban educadores en salud (Claeson \& Waldman, 2000).

15 Resulta significativo en este aspecto la justificación por parte de la APSS de lo innecesario de determinados servicios básicos, como los sistemas de canalización de aguas y de saneamiento.

16 Hay autores que consideran el documento Investing in Health del Banco Mundial como un intento de disminuir la presencia estatal en el sector salud, promoviendo la privatización, haciendo que los países en desarrollo modificaran sus políticas sanitarias si querían seguir recibiendo ayuda de los países donantes (Brown, Cueto \& Fee, 2006; Hall \& Taylor, 2003). 
Sin embargo, lejos de aceptar estos argumentos, el cisma entre ambas posturas y las recriminaciones han ido en aumento. En palabras de Tejada de Rivero:

Lástima que $[. .$.$] por la impaciencia de$ lograr resultados tangibles en lugar de trabajar sobre siempre difíciles procesos de cambio, algunas agencias internacionales, de Naciones Unidas y privadas, contribuyeron a las mayores distorsiones del concepto y la práctica alejadas de la original APS (2003, p. 7).

\section{CONCLUSIONES: EL FRACASO DE ALMA-ATA Y UN FUTURO INCIERTO}

Veintidós años después de la Declaración de Alma-Ata, lejos de cumplirse los objetivos de Salud para Todos, las diferencias entre países del Primero y del Tercer Mundo han aumentado. Alma-Ata ha fracasado en sus objetivos, debido a distintas causas:

- Falta de un auténtico compromiso político.

- Existencia de fondos insuficientes en la mayoría de los casos.

- Aparición de múltiples interpretaciones distintas que minaron su mensaje.

- Fallos metodológicos: decía lo que debía hacerse pero no cómo (Cueto, 2005).

A las que se suman otras teorías, como que subestimó el poder de los hospitales terciarios, de la industria far- macéutica y de los clínicos, a los que no supo convencer. ${ }^{17}$ Chocó con una sociedad cada vez más individualizada y unas políticas económicas basadas en los principios de mercado, centradas en la descentralización y la privatización (Green, Ross \& Mirzoev, 2007). Y, finalmente, Litsios (2002) apunta como causa del fracaso el esfuerzo en la realización de la Conferencia de AlmaAta, lo que minó el potencial de la OMS y de la Unicef de trabajar en forma conjunta en aquellos países con más posibilidades de éxito.

Pese a lo alegado por los defensores de la APSS, la efectividad del modelo de APS ha quedado sobradamente demostrada. Como ejemplos, sirvan las experiencias de Kasongo (Zaire), donde se observó una disminución en la hospitalización debido a la rapidez en el diagnóstico y a una atención más adecuada desarrollada en niveles primarios (Van Lerberghe \& Pangu, 1988).

17 En el caso de la Alianza Mundial para vacunas e inmunización, la existencia de cargos directivos pertenecientes a farmacéuticas ha provocado situaciones cuando menos controvertidas: estudios en Mozambique, Ghana, Lesotho y Tanzania revelan que aunque la cobertura vacunal ha sido ampliada, no se han asegurado recursos adicionales para sus insuficientes sistemas de salud. Otras situaciones han resultado todavía más escandalosas: a Ghana se le dieron diez días para decidir si aceptaba una nueva vacuna para la Hepatitis B sin evidencias científicas de su utilidad. 
El estudio de Hill y colaboradores en Gambia (2000), que valora la acción de los "Village-level health services"18 con una reducción significativa de la mortalidad infantil y una mejora de las condiciones de vida de la población. La experiencia del Caribe (Mullings \& Paul, 2007), que desde principios del siglo XX ha actuado dentro del marco de la APS y que da como resultado la disminución de la mortalidad infantil y una serie de aportaciones a Alma-Ata, como es el énfasis en la promoción, en la prevención y en los programas sociales. Además, están las experiencias de lugares como Bengala, con sus Gonoshasthaya Kendra, que proporcionaban servicios de salud para zonas rurales (Fort, 2006), Cuba, Nicaragua o Bangladesh, donde se trabajaba sobre una base de equidad social (Magnussen, Ehiri \& Jolly, 2004) o Costa Rica y Sri Lanka, con unos indicadores mejor de lo esperado para su desarrollo económico (OMS, 1991).

Si estas experiencias funcionan (y no parecen existir motivos para dudar de ello), ¿por qué seguir insistiendo en programas verticales, cuando podemos llevar a cabo una acción de carácter integral? Parece que todavía no se ha comprendido que la salud es una reali-

18 Ya no solo "intelectualmente" sino también en el plano económico. Los salarios en la APS eran mucho más bajos que los de las agencias que promocionaban programas verticales. Además, se ofrecían primas al personal sanitario para desarrollar programas específicos (Van Balen, 2004). dad social compleja, un deterioro multicausal del individuo en un contexto de adversidad, que por tanto difícilmente mejorará si no abordamos las causas estructurales de la pobreza. El protagonismo de la comunidad que reclama Alma-Ata proporciona a las poblaciones lo más importante: el derecho a elegir su propio futuro, no solo en cuanto al desarrollo de los recursos o la planificación sanitaria, sino también en la capacidad de crear conciencia, promoviendo la responsabilidad personal en salud. ${ }^{19}$ Los programas específicos son necesarios, en cuanto a que siempre existen necesidades concretas, pero no serán factibles en unos sistemas sanitarios apenas desarrollados (Magnussen, Ehiri \& Jolly, 2004).

Sin embargo, y como afirma Cueto: "A culture of survival among the poor sustains the privileges among politicians" (2005, p. 323). ${ }^{20}$ Los programas verticales perpetúan la dependencia de los países en desarrollo, convirtiéndolos en potenciales consumidores, lo que no ha hecho sino aumentar en las últimas décadas con el modelo neolibe-

19 Zonas con un sistema con enfermeras comunitarias, participación de la población y gratuidad en la atención materno-infantil, junto con medidas de desarrollo de la comunidad (transporte, acceso, vivienda) y de fortalecimiento de los servicios de salud.

20 Desarrollado en el White Paper, emitido por el National Health Service (Green, Ross \& Mirzoev, 2007). 
ral y sus consideraciones económicas por encima de las de salubridad.

Pese a que el futuro no resulta muy esperanzador, sigue quedando algún motivo para el optimismo. Iniciativas como los Objetivos de Desarrollo del Milenio indicados en la Declaración para la Salud de los Pueblos del 2005 muestran el compromiso de determinados sectores de la sociedad en la búsqueda de soluciones.

Mahler (1984) dijo:

Too many countries, too many bilateral and multilateral agencies, too many individuals had become too disillusioned with the prospects for genuine human development.

Pero realmente, si de algo hay que culpar a Alma-Ata es de no haber conseguido concientizar a la población y a sus dirigentes de que invertir en salud y en bienestar no solo es el mejor medio de acabar con las desigualdades en salud, sino de conseguir la estabilidad política que tanto preocupa hoy en día (Kleinert, 2002).

\section{REFERENCIAS}

Barriga, S. (1993). La salud ¿Para qué?

En J. León Rubio \& S. Barriga (Comps.). Psicología de la Salud (pp. 17-30). Sevilla: Eudema.

Brown, T. M.; Cueto, M. \& Fee, E. (2006). The World Health Organization and the transition from "International" to "Global" public health. American Journal of Public Health, 96, 62-72.

Claeson, M. \& Waldman, R. (2000). The evolution of child health programmes in developing countries: from targeting diseases to targeting people. Bulletin of the World Health Organization, 78, 1234-45.

Cueto, M. (2004). The origins of primary health care and selective primary health care. American Journal of Public Health, 94, 1864-74.

Cueto, M. (2005). The promise of primary health care. Bulletin of the World Health Organization, 83, 322-23.

De Maeseneer, J.; Willems, S.; De Sutter, A.; Van de Geuchte, I. \& Billings, M. (2007). Primary health care as a strategy for achieving equitable care: a literature review commissioned by the Health Systems Knowledge Network. Recuperado el 3 de abril de 2009, de http://www.who.int/social_determinants/resources/csdh_media/primary_health_care_2007_en.pdf

Fleck, F. (2002). Children's charity criticises global immunisation initiative. British Medical Journal, 324, 129.

Fort, M.; Mercer, M. A. \& Gish, O. (2006). El negocio de la Salud: Los intereses de las multinacionales y la privatización de un bien público. Barcelona: Paidós. 
Gervas, J.; Pérez-Fernández, M.; Palomo-Cobos, L. \& Pastor-Sánchez, R. (2005). Veinte años de reforma de la Atención Primaria en España. Valoración para un aprendizaje por acierto / error. Recuperado el 26 de noviembre de 2007, de www.msc.es

Green, A.; Ross, D. \& Mirzoev, T. (2007). Primary Health Care and England: The coming of age of Alma-Ata? Health Policy, 80, 11-31.

Hall, J. \& Taylor, R. (2003). Health for all beyond 2000: the demise of the Alma-Ata declaration and primary health care in developing countries. Medical Journal of Australia, 178, 17-20.

Hill, A. G.; MacLeod, W. B.; Joof, D.; Gomez, P.; Ratcliffe, A. A. \& Walraben, G. (2000). Decline of mortality in children in rural Gambia: the influence of village-level Primary Health Care. Tropical Medicine and International Health, 5, 107-18.

Kleinert, S. (2002). What are the world's priorities? The Lancet, 360, 1118.

Litsios, S. (2002). The long and difficult road to Alma-Ata: a personal reflection. International Journal of Health Services, 32, 709-732.

Litsios, S. (2004). The Christian Medical Commission and the Development of the World Health Organization's Primary Health Care Ap- proach. American Journal of Public Health, 94, 1884-1893.

Magnussen, L.; Ehiri, J. \& Jolly, P. (2004). Comprehensive versus Selective Primary Health Care: lessons for global health policy. Health affairs, 23, 167-76.

Mahler, H. T. (1975). Social perspectives in health. Discurso en la $29^{\mathrm{a}}$ asamblea de la OMS. Mayo, Ginebra.

Mahler, H. T. (1978). Promotion of Primary Health Care in Member Countries of WHO. Public Health Report, 93, 107-113.

Mahler, H. T. (1984). World Health 2000 and Beyond. Discurso en la $41^{\text {a }}$ asamblea de la OMS. Mayo, Ginebra.

Mullings, J. \& Paul, T. J. (2007). Health sector challenges and responses beyond the Alma-Ata Declaration: a Caribbean perspective. Pan American Journal of Public Health, 21, 155-63.

Newell, K. (1988). Selective Primary Health Care: the Counter Revolution. Social Science Medicine, 26, 903-906.

OMS. (1978). Declaration of AlmaAta. [Versión electrónica]. Recuperado el 3 de abril del 2009, de www.who.int/hpr/NPH/docs/ declaration almaata.pdf. 
OMS. (1991). De Alma-Ata al año 2000. Reflexiones a medio camino. Ginebra: OMS.

Tejada de Rivero, A. (2003). AlmaAta: 25 años después. [Versión electrónica] Perspectives in Health, 8. Recuperado el 3 de abril de 2009, de la base de datos de la Organización Panamericana de la Salud.

Van Balen, H. (2004). Disease control in primary health care: a historical perspective. Tropical Medicine and International Health, 9, 22-26.

Van Lerberghe, W. \& Pangu, K. (1988). Comprehensive can be effective: the influence of coverage with a health centre network on the hospitalization patterns in the rural area of Kasongo, Zaire. Social Science Medicine, 26, 949-55.

Walsh, J. \& Warren, K. (1979). Selective Primary Health Care: an interim strategy for disease control in developing countries. New England Journal of Medicine, 301, 967-974.

Warren, K. (1988). The evolution of selective primary health care. Social Science Medicine, 26, 891-98.

World Bank. (1993). World development report 1993: investing in health. Nueva York: Oxford University Press. 